'Alumno de Medicina de la Pontificia Universidad Católica Argentina.

${ }^{2}$ Servicio Clínica Médica, Hospital Británico de Buenos Aires, Argentina y Profesor Titular de Historia de la Medicina de la Pontificia Universidad Católica Argentina

Recibido el 17 de junio de 2014, aceptado el 28 de agosto de 2014. Correspondencia a: Pablo Young Hospital Británico. Perdriel 74 (1280) Buenos Aires, Argentina. Tel 541143096400 Fax 541143043393 pabloyoung2003@yahoo.com.ar

\section{Perspectiva histórica}

En la década de 1930, el laboratorio de fisiología de la Facultad de Medicina de la Universidad de Buenos Aires, dirigido por el premio Nobel Bernardo Houssay (1887-1971), viviría uno de sus períodos más prolíficos.

Seis hombres, incluyendo al propio Houssay, se encaminarían en el laborioso e intricado camino del estudio de la hipertensión arterial nefrogénica.

\section{Investigaciones previas que sirvieron de sustento para el equipo argentino}

En 1898, Robert Tigerstedt (1853-1923) (Figura 1) y su estudiante Gunner Bergman, publicaron un trabajo denominado "El riñón y la circulación". En el mismo mostraron la función presora de una sustancia a la que denominaron renina, esta era obtenida a partir de extractos de corteza renal ${ }^{1}$. Es todavía un enigma por qué Tigerstedt abarcó este tema, una de las explicaciones a este interrogante pudo ser el vínculo establecido anteriormente por Richard Bright (1789-1859) entre la enfermedad renal y la hipertensión o el descubrimiento por parte de Charles Édouard Brown Séquard (18171894) de una hormona suprarrenal, como así también, otras versiones afirman que lo habría hecho porque necesitaba trabajos para publicar en el Congreso Internacional de Medicina, que se llevó a cabo en la ciudad de Moscú en $1897^{2}$. Regresó a Finlandia desde Estocolmo, donde había realizado la investigación en el Instituto Karolinska, y no prosiguió con ella ${ }^{3}$.

Muchos intentos se habían realizado para crear un modelo experimental de hipertensión arterial, como irradiar el parénquima renal, reducir su masa mediante ablaciones, la oclusión de ramas de la arteria renal, sin embargo, estos no fueron exitosos. En 1909, Theodore Caldwell Janeway (1872-1917) describió un aumento de la tensión arterial luego de la oclusión de ramas de la arteria 


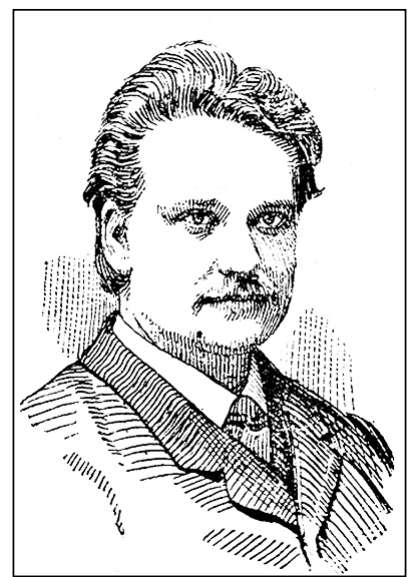

Figura 1. Dr. Robert Tigerstedt. http://commons.wikimedia.org/wiki/ File:Robert Tigerstedt.jpg; consultado 5/4/2014.

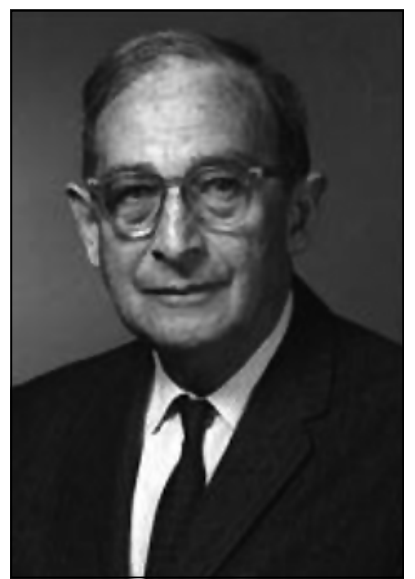

Figura 2. Dr. Harry Goldblatt. http://artsci.case. edu/dittrick/online-exhibits/explore-the-artifacts/ goldblatt-clamps-1934/; consultado 19/04/2014.

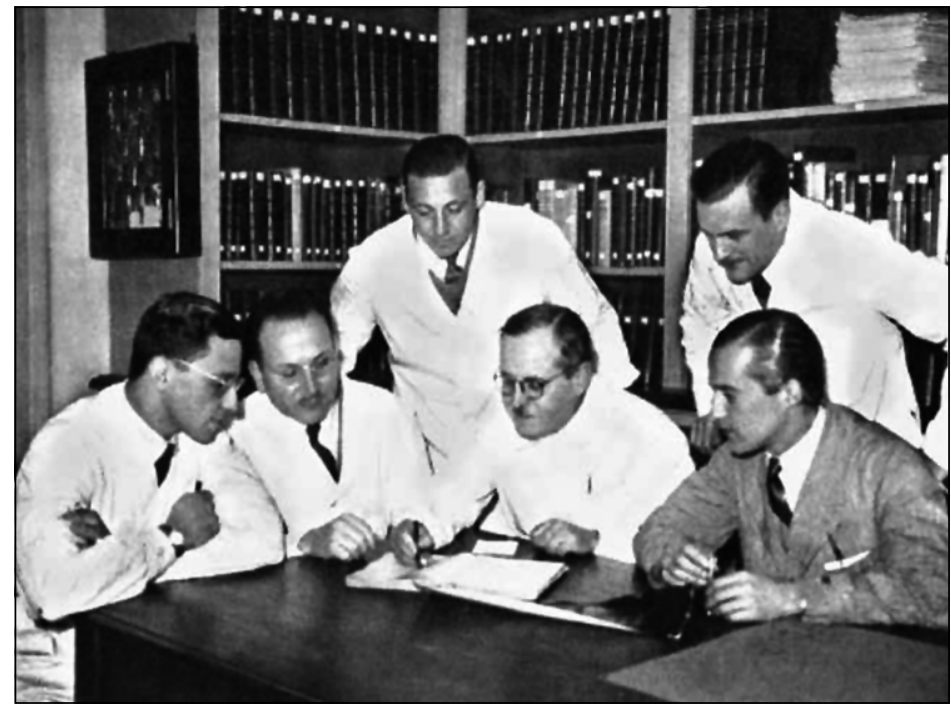

Figura 3. Los integrantes del grupo fueron de izquierda a derecha Fasciolo, Muñoz, Taquini, Houssay, Braun Menéndez y Leloir. En: http:// www.scielo.org.ar/scielo.php?script $=$ sci ar ttext\&pid =S0025-76802004000200015; consultado 19/04/2014. renal y ablación del riñón contralateral, su experiencia no tuvo mucha repercusión ${ }^{4}$.

No fue hasta 1934, cuando Harry Goldblatt (1891-1977) (Figura 2) creó un modelo experimental de hipertensión arterial, mediante la obstrucción de la arteria renal ${ }^{5}$, conocido como riñón de Goldblatt. El mismo dedujo esto a partir de las estrecheces que observó en arterias intrarenales y no en la misma arteria renal. Alberto Agrest (1923-2012) señaló que es llamativo que un patólogo como Goldblatt no haya observado anteriormente la asociación entre estrecheces de la arteria renal e hipertensión ${ }^{6}$.

\section{Trabajo realizado en el Instituto de Fisiología, dirigido por el doctor Bernardo Houssay}

En el año 1934, Juan Carlos Fasciolo (19111993) (Figura 3), estudiante de la Carrera de Medicina de la Universidad de Buenos Aires, debió presentar una tesis para obtener su título de grado. El doctor Houssay le sugirió que investigue acerca de la hipertensión arterial nefrogénica, la inclinación del mismo hacia este tema estaba dada por el fallecimiento prematuro de su discípulo Juan Guglielmetti (1891-1922) de hipertensión arterial maligna ${ }^{7}$. 
Tabla 1. Cronología de los avances en el sistema renina-angiotensina-aldosterona

\begin{tabular}{|c|c|c|c|}
\hline Año & Autores & Descubrimiento & Referencia \\
\hline 1898 & Tigerstedt R, Bergman G. & Función presora de la renina, extraída de la corteza renal & 1 \\
\hline 1909 & Janeway TC. & $\begin{array}{l}\text { Aumento de la presión arterial mediante oclusión de } \\
\text { ramas de la arteria renal y nefrectomía contralateral }\end{array}$ & 4 \\
\hline 1931 & Volhard F. & Sugiere mecanismo humoral en la hipertensión arterial & 10 \\
\hline 1934 & Goldblatt H. & $\begin{array}{l}\text { Modelo experimental de hipertensión arterial mediante } \\
\text { oclusión de la arteria renal }\end{array}$ & 5 \\
\hline 1937 & Fasciolo JC, Houssay BA. & $\begin{array}{l}\text { Aumento de la tensión arterial en perros tras el implante } \\
\text { a nivel cervical de riñones isquémicos }\end{array}$ & 9 \\
\hline 1938 & Kohlstaedt KG, Helmer OM, Page IH. & Activación de la renina por un componente del plasma. & 25 \\
\hline 1938 & Taquini AC, Houssay BA. & $\begin{array}{l}\text { Acción vasoconstrictora directa de preparados de plasma } \\
\text { proveniente de la vena renal de perros hipertensos }\end{array}$ & 16 \\
\hline 1939 & Braun Menéndez E, Fasciolo JC. & $\begin{array}{l}\text { Liberación de sustancia vasoconstrictora en isquemia } \\
\text { aguda. }\end{array}$ & 18 \\
\hline 1939 & $\begin{array}{l}\text { Braun-Menéndez E, Fasciolo JC, } \\
\text { Leloir LF, Muñoz JM. }\end{array}$ & $\begin{array}{l}\text { Se aisló la hipertensina y se propone un mecanismo } \\
\text { enzimático para su formación }\end{array}$ & 21 \\
\hline 1940 & Page IH, Helmer OM. & $\begin{array}{l}\text { Aíslan la angiotonina, proponen un mecanismo } \\
\text { enzimático, donde la renina es la enzima y el activador de } \\
\text { la renina el sustrato }\end{array}$ & 27 \\
\hline 1958 & Page IH, Braun Menéndez E. & Acuerdan nueva nomenclatura & 29 \\
\hline 1975 & Ondetti MA & Descubrimiento del Captopril & 30 \\
\hline
\end{tabular}

Fasciolo en un principio buscó destruir la corteza renal de ratas para contar con un modelo de hipertensión arterial experimental, sin embargo, encontró dificultades en el método y los resultados no fueron congruentes. Leyó el artículo publicado por Goldblatt, y comenzó a aplicar el método descripto por éste en perros ${ }^{8}$. En el año 1937, utilizando una técnica que le fuera enseñada por su maestro, implantó el riñón isquémico de perros hipertensos en perros normotensos nefrectomizados, lo que provocó un aumento de la presión arterial ${ }^{9}$. Manifestó que utilizó esta técnica, porque supuso que en el riñón se producía constantemente una sustancia hipertensora y que la misma era metabolizada de manera muy rápida, por lo que no podía ser detectada ${ }^{8}$. Esta fue la primer experiencia que permitió confirmar la presencia de un mecanismo humoral como responsable de la hipertensión arterial nefrogénica. Recordemos que anteriormente esto había sido sugerido por Volhard y Goldblatt, a pesar de esto, las experiencias previas llevadas a cabo por el médico argentino no habían alcanzado resultados positivos $^{10-12}$. El papel del riñón contralateral y de la glándula suprarrenal en la hipertensión arterial provocada por isquemia renal, también fueron estudiados por Fasciolo ${ }^{13,14}$.

Alberto Carlos Taquini (1905-1998), que en el año 1931 tuvo el privilegio de escuchar personalmente a Franz Volhard (1872-1950), postulando la teoría humoral acerca del vasoespasmo implicado en la hipertensión arterial, discutió con Fasciolo y Houssay la posibilidad de que la sustancia liberada por el riñón actuara directamente sobre los vasos sanguíneos, estos apoyaron su idea y el comprobó esto utilizando plasma proveniente de la sangre venosa de riñones isquémicos, utilizando la técnica de Loewen Trendelenburg en $1938^{15,16}$. También, durante el mismo año, Taquini logró demostrar que el aumento de la presión arterial que se veía luego de restituir el flujo en riñones isquémicos era provocado por la misma sustancia vasopresora involucrada en los estudios anteriores ${ }^{17}$.

Fasciolo junto a Eduardo Braun Menéndez (1903-1959), que había vuelto de una beca en Inglaterra, donde trabajo con el doctor Charles 
Arthur Lovatt Evans (1884-1968) estudiando el metabolismo del corazón, mostraron mediante la utilización de un preparado corazón-pulmón y la perfusión de un riñón aislado que la interrupción del flujo durante unos pocos minutos ya provocaba la presencia de la sustancia hipertensora en la sangre venosa del riñón, esto comprobado mediante la inyección de la misma a perros nefrectomizados. Más adelante, lo antes señalado cobraría gran importancia, ya que para poder aislar el principio causante del efecto hipertensor se requerirían grandes cantidades de sangre venosa que contengan el mismo y la preparación de perros hipertensos no era sencilla ${ }^{8,18}$.

En base a los avances alcanzados y la elevada importancia que había cobrado el tema, Houssay con gran sabiduría, formó un equipo para tratar de aislar la sustancia en cuestión. El mismo estuvo formado por Braun Menéndez, Luis Federico Leloir (1906-1987), Juan M. Muñoz y Fasciolo (Figura 3). Taquini había partido hacia Estados Unidos de Norteamérica para trabajar en Harvard junto a los doctores David B. Dill (1891-1986) y Paul Dudley White (1886-1973) ${ }^{19}$. Este grupo, quizás uno de los más brillantes que la ciencia Argentina ha tenido, funcionaba en total armonía, en palabras de Leloir, quien resaltó "el buen ánimo que reinaba en el laboratorio" y Fasciolo quien señaló la importancia de la diversidad del conocimiento de los cuatro, al manifestar que "Leloir y Muñoz estaban más avocados a la bioquímica mientras que Braun Menéndez y él a la fisiología"20.

En 1939, hallaron la sustancia hipertensora a partir de sangre venosa de riñones sometidos a períodos de isquemia, la misma era extraída mediante acetona. Esta producía un aumento de la tensión arterial cuando era inyectada en animales, sin embargo, el efecto duraba sólo unos minutos, un escenario muy diferente al que se obtenía mediante el implante de riñones isquémicos en la circulación cervical, donde el aumento de la tensión arterial era de carácter prolongado. La sustancia aislada era termoestable, dializable y tenía un efecto hipertensor breve, características que la diferenciaban de la renina. El equipo argentino denominó a esta sustancia hipertensina. El siguiente paso fue dilucidar la relación existente entre la renina y la hipertensina. En primera instancia se incubaron fragmentos de corteza renal con plasma en condiciones de anoxia, sin embargo, no se pudo hallar a la hipertensina, posteriormente se manifestó que esto se debía a la presencia de enzimas que metabolizaban la misma. En un segundo intento, se incubaron extractos de renina con plasma a una temperatura de $37^{\circ} \mathrm{C}$, pudiendo de esta manera obtener la sustancia hipertensora in vitro. Basándose en lo antes expuesto, se postuló una reacción enzima-sustrato para la formación de hipertensina, donde se denominó hipertensinógeno al sustrato, renina a la enzima e hipertensinasas a las enzimas encargadas de degradar a la hipertensina ${ }^{21,22}$.

Este notable descubrimiento permitió también comprender mejor las experiencias realizadas previamente. El efecto hipertensor que se lograba mediante el implante de riñones isquémicos, era de carácter prolongado, y estaría dado por la liberación de renina y la continua formación de hipertensina en el plasma.

El descubrimiento de Braun Méndez y su equipo se limitó a la angiotensina. Por esos años no se mencionaba aún a la aldosterona, la que en los años 40 no se conocía en sus detalles fisiológicos ni era parte del sistema renina angiotensina aldosterona. En ese tiempo se conoció un potente mineralocorticoide denominado electrocortina por Grundy. En 1953, la inglesa nacida en Rusia Sylvia Agnes Sophia Tait (1917-2003) y cols. indentificaron la electrocortina mediante cromatografía. La hormona se rebautizó aldosterona. En 1955, Jerome W. Conn (1907-1994) describió el hiperaldosteronismo primario, producido por un adenoma suprarrenal único.

Así como Braun Méndez era chileno, hubo un importante contacto del grupo argentino con científicos chilenos, como el Dr. Héctor Croxatto Rezzio (1908-2010), quien descubrió otros péptidos vasoactivos que provenían de la hidrólisis de proteínas plasmáticas.

Unido a los estudios in vivo con los perros, por esos años se popularizaron los bio-ensayos de angiotensina y de los péptidos vasoactivos, que aclararon el rol de angiotensina en el control de la resistencia periférica, además de su efecto cardiaco y sobre los terminales nerviosos simpáticos.

Esta investigación se grafica magistralmente por una frase que se atribuye a Isaac Newton "He podido ver el más allá por estar parado sobre las espaldas de un gigante". Este gigante representa el acúmulo del saber y hoy, metafóricamente, para llegar a un descubrimiento -el breakthroughhay que "escalar por la espalda del gigante hasta 
llegar a sus hombros"; la dificultad de la escalada es proporcional al aumento del saber, y como el progreso no se detiene se tardará cada vez más en alcanzar la cima ${ }^{23}$.

Es importante destacar que Taquini no estaba en la Argentina en el momento en que se logró aislar la hipertensina, y aludiendo a esta situación el mismo manifestó, "Erróneo en el tiempo como diría Borges, cuando ellos llegaron a identificarla yo estaba en Harvard"15. También en un libro publicado por Irvine H. Page (1901-1991), este manifestó su asombro al ver que Taquini no estaba incluido en el trabajo donde se expuso el descubrimiento ${ }^{24}$.

\section{Simultáneamente en Estados Unidos de Norteamérica se alcanzaban las mismas conclusiones}

Page, K G. Kohlstaedt y O M. Helmer conformaban el equipo norteamericano que estuvo involucrado en el esclarecimiento de lo que hoy se conoce como sistema renina-angiotensinaaldosterona.

El enfoque de los mismos fue diferente al utilizado por el grupo argentino. Los estadounidenses se dedicaron a concentrar la renina a partir de extractos de parénquima renal y estudiar su función vasoconstrictora en preparados de cola de perro y oreja de conejo. En estas experiencias comprobaron que la vasoconstricción sólo se daba cuando el preparado se encontraba perfundido con suero y no así cuando se utilizaba Ringer lactato, lo que los llevó en el año 1938, a pensar que en el suero se encontraba una sustancia activadora de la reni$\mathrm{na}^{25}$. En el año 1939, esta conclusión fue expuesta por Page y cols. en una reunión de la American Heart Association, Taquini que se encontraba en el auditorio y estando al tanto de los avances del equipo argentino, refutó lo expuesto por Page diciendo que no era la renina activada la sustancia que provocaba la vasoconstricción sino una sustancia totalmente diferente ${ }^{15}$. Page manifestó que fue muy criticado por haber utilizado la palabra activador de la renina, pero que lo hizo queriendo ser prudente, ya que la reacción enzimática catalizada por la renina no había sido demostrada en ese momento ${ }^{26}$. En el año 1940, el equipo aisló a la angiotonina, equivalente de la hipertensina del grupo argentino, esta era obtenida por la interac- ción entre el activador de la renina, sustrato y la renina, enzima ${ }^{27}$. Luego Page y cols. revisaron la denominación llamado al activador de la renina, hipertensinógeno o sustrato de la renina ${ }^{28}$.

\section{Un final de caballeros}

Braun Menéndez y cols. debieron estar muy desilusionados cuando, a los pocos meses de su publicación, Page y su equipo alcanzó los mismos resultados utilizando otro camino. Lejos del conflicto, en el año 1958, Braun Menéndez y Page acordaron en Ann Arbor una nueva nomenclatura, de esta manera surgieron las palabras angiotensina y angiotensinógeno que se obtuvieron de la combinación de los nombres antes establecidos por ambos ${ }^{26,29}$. En el año 1985, Page le envió una carta a Fasciolo, manifestando que esperaba que la manera en que se resolvió este conflicto sirva de ejemplo a las generaciones de científicos venideras ${ }^{8}$.

\section{Proyección traslacional}

Al decir de Houssay, la fisiología da lugar al "arte de curar con bases científicas" que se ve reflejado en este descubrimiento a su aplicación práctica, particularmente con la contribución de otro argentino, el químico Miguel Angel Ondetti, (1930-2004) quien sintetizó el captopril en 1975, el primero de los inhibidores de la enzima convertidora de angiotensina, precursor del enalapril y otros, que han sido de gran importancia terapéutica y que han demostrado finalmente la relevancia de la angiotensina en el control cardiovascular ${ }^{30}$.

\section{Lo que estos grandes hombres propulsaron en la Argentina}

Houssay no tuvo «maestro», fue un autodidacta, lo que de por sí lo marca como investigador excepcional. Nacido en 1887 y Bachiller a los 13 años, ingresó en 1900 a la entonces Escuela de Farmacia de la Universidad de Buenos Aires ${ }^{31}$. Se recibió de Farmacéutico a los 17 años e ingresó a la Facultad de Medicina para cumplir su verdadera vocación. Médico a los 23 años, en 1910, con muy altas calificaciones, la lectura de la Introducción 
a la Medicina Experimental de Claude Bernard lo decidió tempranamente por la investigación fisiológica. Cuando en 1919 fue nombrado Profesor Titular de Fisiología y Director del Instituto de Fisiología de la Facultad de Medicina de la Universidad de Buenos Aires, se consagró enteramente a la docencia y a la investigación, cumpliendo religiosamente el full-time que preconizó durante toda su vida. Durante casi un cuarto de siglo (1919-1943) trabajó extraordinariamente, impartiendo enseñanza teórica y práctica a los alumnos de Medicina, Odontología y Farmacia. Abrió una variedad de campos de investigación, formando numerosos discípulos a los que enviaba luego a perfeccionarse al exterior con «obligación» de volver al país ${ }^{31,32}$. Entre los logros más grandes, además del Nobel que le fue otorgado por sus trabajos de la influencia del lóbulo anterior de la hipófisis en la distribución de la glucosa en el cuerpo, en 1934 creó la Asociación Argentina para el Progreso de las Ciencias (AAPC); el 8 de abril de 1944 fundó el Instituto de Biología y Medicina Experimental (IBYME), seguido al poco tiempo del Instituto de Investigaciones Bioquímicas (IIB), producto del apoyo de la familia Campomar y que dirigió desde sus inicios Luis Federico Leloir. En febrero de 1958 fundó el Consejo Nacional de Investigaciones Científicas y Técnicas (CONICET), lo que significó el mayor logro para la investigación argentina; pero posiblemente el más importante, haya sido ser la luz que iluminó el camino de la ciencia médica argentina a través de sus discípulos ${ }^{33-35}$. No se puede olvidar que, además de las instituciones citadas, fue el creador de la Asociación Latinoamericana de Ciencias Fisiológicas y el Comité Interamericano de Ciencia y Tecnología de la Organización de Estados Americanos $^{31}$.

Algunos de sus discípulos fueron Froilán P. Ludueña (1906-1982), Oscar Orias (1905-1955), Venancio Deulofeu (1902-1984), Virgilio G. Foglia (1905-1993), Juan T. Lewis (1898-1976), Muñoz, Fasciolo, Taquini, y Leloir que era francés y por adopción argentino, fue premio Nobel de Química por su descubrimiento sobre la síntesis de carbohidratos $^{32}$. Braun Menéndez fue chileno y naturalizado argentino a temprana edad, estudió en la Universidad de Buenos Aires e investigó bajo la tutela de Houssay. Taquini, junto a otros hombres del momento, fundó en 1960 la Sociedad Argentina de Investigación Clínica (SAIC) que reúne hoy día los mejores investigadores clínicos de la Argentina ${ }^{34}$. Alfredo Lanari (1910-1985) fundó en 1939 ,junto a otros colegas, la prestigiosa Revista Medicina, a cuyo cuerpo editorial perteneció hasta su muerte, y fundó en 1958 el Instituto de Investigaciones Médicas de la Universidad de Buenos Aires, que luego llevaría su nombre ${ }^{35-37}$.

El descubrimiento del sistema renina-angiotensina-aldosterona es mucho más que un conocimiento teórico obligatorio para cualquier libro de fisiología. Sin lugar a dudas representa uno de los puntos culmines de la fisiología argentina y lo que es aún más importante, una muestra del camino que debe tomar la ciencia, construyendo sobre los avances realizados por otros colegas y situando a la búsqueda de la verdad como punto cardinal.

Agradecimientos: Agradecemos a la Dra. Christiane Dosne Pasqualini por la revisión crítica del manuscrito.

\section{Referencias}

1. Tigerstedt R, Bergman PG. Niere und Kreislauf. Skand Arch Physiol 1898; 8: 223-71.

2. Zylberman M, Young P. Enfermedad pericárdica: de la antigüedad al ultrasonido. Gac Med Mex 2010; 146: 348-53.

3. Hall JE. Historical Perspective of the Renin Angiotensin System. En: H. Wang, Angiotensin Protocols. Totowa, New Yersey: Editorial Humana Press Inc.; 2001. p. 3-5.

4. Janeway TC. Note on the blood pressure changes following reduction of the renal arterial circulation. Proc Soc Exp Biol Med 1909; 109: 5-6.

5. Goldblatt H, Lynch J, Hanzal RF, Summerville WW. Studies on experimental hypertension, I: the production of persistent elevation of systolic blood pressure by means of renal ischemia. J Exp Med 1934; 59: 347-79.

6. Agrest A. La saga de la renina. Medicina (B Aires) 2000; 60: 37-45.

7. Milei J, Trujillo JM. Historia del Instituto de investigaciones cardiológicas Alberto C. Taquini en su $60^{\circ}$ aniversario. Medicina (B Aires) 2004; 64: 163-9.

8. Fasciolo JC. Argentina. The experimental observation that led to discovery of angiotensin. 1939 Buenos Aires, Argentina. Hypertension 1990; 16: 194-8.

9. Houssay BA, Fasciolo JC. Demostración del mecanismo humoral de la hipertensión nefrógena. Bol Acad Nac Med 1937; 18: 342-4.

10. Volhard F. Nieren und ableitende Harnwege. In: Bergmann G, Staehelin R, eds. Handbuch der Inneren Medi- 
zin, vol 6. Berlin: Springer Verlag 1931: 1-1023.

11. Harrison TR, Blalock A, Mason MF. Effect on blood pressure of injection of kidney extracts of dogs with renal hypertension. Proc Soc Exp Biol 1937; 35: 38-40.

12. Prinzmetal M, Friedman B. Pressor effects of kidney extracts from patients and dogs with hypertension. Proc Soc Exp Biol 1937; 35: 122-4.

13. Fasciolo JC. Acción del riñón sano sobre la hipertensión arterial por isquemia renal. Rev Soc Argent 1938; 14: 15-24.

14. Fasciolo JC. Papel de las glándulas adrenales en la génesis de la hipertensión arterial por isquemia renal. Rev Soc Argent 1938; 14: 25-30.

15. Milei J. Alberto C. Taquini and the 'links' that led to the discovery of angiotensin: on the 100th anniversary of his birth. J Hypertens 2005; 23: 1267-9.

16. Houssay BA, Taquini AC. Acción vasoconstrictora de la sangre venosa del riñón isquemiado. Rev Soc Arg Biol 1938; 14: 5-14.

17. Taquini AC, Braun-Menéndez E. Liberación de substancia vasoconstrictora en el riñón completamente isquemiado. Rev Soc Arg Biol 1938; 14: 422-9.

18. Braun-Menéndez E, Fasciolo JC. Acción vasoconstrictora e hipertensora de la sangre venosa del riñón en isquemia incompleta aguda. Rev Soc Arg Biol 1939; 15: 161-72.

19. Basso N, Schiffrin L. Professor Alberto C. Taquini: 19051998. Hypertension 1998; 32: 1-2.

20. Pasqualini CD. Juan Carlos Fasciolo, discípulo de Houssay y descubridor de la angiotensina. Medicina (B Aires) 2011; 71: 405-7.

21. Braun-Menéndez E, Fasciolo JC, Leloir LF, Muñoz JM. La sustancia hipertensora de la sangre del riñón isquemiado. Rev Soc Arg Biol 1939; 15: 420-5.

22. Braun-Menéndez E, Fasciolo JC, Leloir LF, Muñoz JM. The substance causing renal hypertension. J Physiol 1940; 98: 283-98.

23. Pasqualini CD. El destino de los investigadores en ciencia. Medicina (B Aires) 2013; 73: 179-82.
24. Page IH. Hypertension research: a memoir. Elmsford, NY: Pergamon Press; 1988, p. 116.

25. Kohlstaedt KG, Helmer OM, Page IH. Activation of rennin by blood colloids. Proc Soc Exp Biol Med 1938; 39: 214-5.

26. Page IH. Hypertension research. A memoir 1920-1960. Hypertension 1990; 16: 199-200.

27. Page IH, Helmer OM. A crystalline pressor substance (angiotonin) resulting from the reaction between renin and renin-activator. Exp Med 1940; 71: 29-42.

28. Page IH, Helmer OM, Plentl AA, Kohlstaedt KG, Corcoran AC. Suggested change in designation of "reninactivator" (hypertensinogen) to "renin-substrate (a2 globulin)”. Science 1943; 98: 153.

29. Braun-Menéndez E, Page IH. Suggested revision of nomenclature: angiotensin. Science 1958; 127: 242.

30. Ondetti MA. From peptides to peptidases: a chronicle of drug discovery. Annu Rev Pharmacol Toxicol 1994; 34: 1-16.

31. Agüero AL, Sanchez NI, Cabrera Fischer EI. La organización científica y tecnológica en la Argentina en los tiempos de Bernardo A Houssay y sus primeros becarios. Buenos Aires: Editorial Letra Viva.; 2009. p. 5-119.

32. Pasqualini CD. Bernardo A. Houssay (1887-1971). Medicina (B Aires) 2003; 63: 90-1.

33. Pasqualini CD. 1944 - Houssay y el nacimiento del IBYME, Instituto de Biología y Medicina Experimental. Medicina (B Aires) 2014; 74: 216-9.

34. Pasqualini CD. El significado del Premio Nobel en Fisiología o Medicina. Medicina (B Aires) 2007; 67: 309-13.

35. Pasqualini CD. Taquini y la Sociedad Argentina de Investigación Clínica. En: Alberto C. Taquini y el 75º Aniversario del descubrimiento de la Angiotensina. José Milei y col. Buenos Aires: ININCA, 2014; 77-86.

36. Pasqualini CD. Mis primeros contactos con nuestra revista. Medicina (B Aires) 2010; 70: 96-99.

37. Young P, Finn BC, Bruetman JE, Young DR. Alfredo Lanari, su historia y la metodología de la investigación. Revista Fronteras en Medicina 2009; 4: 38-47. 